\title{
Zur meteorologischen Interpretation der pleistozänen Klimaschwankungen
}

\author{
Von Hermann Flohn, Bonn
}

\begin{abstract}
$\mathrm{Zusammenfassung:} \mathrm{Einige} \mathrm{allgemeine} \mathrm{Fragen} \mathrm{der} \mathrm{pleistozänen} \mathrm{Klimaschwankungen}$ werden vom meteorologischen Standpunkt aus erörtert, unter besonderer Berücksichtigung des Massen- und Wärmehaushalts. Hierzu gehören

das Auftreten alternierender Pluviale von entweder tropischer oder polarer Herkunft, von denen nur die letzteren mit den Eiszeiten synchronisieren,

die Deutung des allmählichen Absinkens der interglazialen Meeresstände als Folge des graduellen Aufbaus des antarktischen Inlandeises seit Beginn des Pleistozäns,

die Diskussion der meteorologischen Verhältnisse während der postglazialen Abschmelzperiode der kontinentalen Inlandeismassen.

$\mathrm{Summary}$ : Some general problems of the Pleistocene climatic fluctuations are discussed from a meteorological view-point, with special reference to the mass and heat budget. Among these are:

the occurrence of alternating pluvial epochs, either of tropical or of polar origin, where only the latter can be synchronized with the glacial epochs,

the hypothesis of a steady lowering of the interglacial ocean levels as caused by the gradual building-up of the antarctic ice-cap since the early Pleistocene,

the discussion of the meteorological conditions during the Postglacial melting period of the continental ice-caps.
\end{abstract}

\section{Einleitung}

Das in der Geologie so oft bewährte Aktualitätsprinzip spielt auch in der Diskussion der Klimaschwankungen der geologischen Vorzeiten eine wesentliche Rolle. Wenn man aus der geographischen Verteilung der Inlandeisgebiete und Gebirgsvergletscherungen auf die sie erzeugenden meteorologisch-klimatologischen Vorgänge schließen will, so gelangt man zu der Vorstellung (Flohn 1952), daß die Klimaanomalien der Zeit des Aufbaues und des Hochstandes der jüngsten Eiszeit eine bemerkenswerte Parallele zu den Witterungsvorgängen in den strengen Wintern Europas aufweisen. Bei dieser meteorologisch-klimatologischen Interpretation der geologischen Befunde muß allerdings mit Vorsicht vorgegangen werden, um die sich regional ganz verschieden auswirkenden Großwettertypen nicht zu verwechseln. Wenn wir die hemisphärische Klassifikation von H. C. WILLETt in ihrer revidierten Form (WILIETT \& SANDERS 1959) übernehmen, dann handelt es sich bei dieser Phase nicht so sehr um eine reine „low-index-Zirkulation“, sondern um eine „highindex-Zirkulation in niedrigen Breiten ". Hierbei kommt es in mittleren Breiten (40-60') zur meridionalen Aufspaltung der troposphärischen Westdrift in blockierende Hochs und quasistationäre Tiefs, die sich bei $300 \mathrm{mb}$ Druck (entsprechend etwa $9 \mathrm{~km}$ Höhe) am deutlichsten ausprägen. Gleichzeitig wird aber auch die hochtroposphärische zonale Westdrift in subtropischen Breiten verstärkt und konzentriert, besonders durch die thermische Wirkung der kontinentalen Inlandeismassen in Nordamerika und Europa. Eine klimatologische Interpretation der Verhältnisse am Südrande der beiden Inlandeis-Schilde führt notwendig (s. auch WILLETT 1950) zur Vorstellung einer sehr intensiven, west-ost verlaufenden Frontalzone in etwa $40^{\circ}$ Breite im mittleren und östlichen Nordamerika, in etwa $45^{\circ}$ Breite im europäischen Mittelmeer- und Schwarzmeergebiet. Das bedeutet in diesem Bereich häufige und intensive Niederschläge, vorwiegend starke Bewölkung und kühle Temperaturen über das ganze Jahr hinweg, eine gewisse Fixierung der „Polarfront" (und der mit ihr gekoppelten Strahlströme) in relativ geringem Abstand von der subtropischen Strahlströmung.

Eine solche aktualistische Interpretation - auf deren physikalisch-meteorologische Be- 
gründung hier nicht eingegangen werden soll - könnte regional und zeitlich noch weiter geführt werden. Aber sie hat ihre Grenzen: es gibt einwandfreie geologische Befunde, deren meteorologische Interpretation nicht mehr durch Heranziehung aktualistischer Erfahrungen erleichtert wird. Das nächstliegende Beispiel ist das der „akryogenen“ Polarklimate mit einem eisfreien Polargebiet (Ввоокs 1949), für das es keine aktuelle Analogie gibt. Für ein solches eisfreies Polarklima existieren in den Interglazialzeiten im Bereich des arktischen Ozeans schlüssige Belege, die anscheinend auch für die postglaziale Wärmezeit beweiskräftig sind. Im Gegensatz hierzu war der antarktische Kontinent sicher in allen pleistozänen Interglazialzeiten ständig vereist gewesen (s. Abschnitt III). Wenn nun auf dem gesamten arktischen Ozean die meeresnahe I.uftemperatur im Polarsommer zu etwa $+5^{\circ} \mathrm{C}$ statt um $0^{\circ}$, im Winter $\mathrm{zu}-1^{\circ}$ statt um $-30^{\circ}$ angenommen werden muß und wenn die Albedo von heute 30-50\% im Sommer, 50-80\% im Winter auf 2-5\% absinkt, dann ergeben sich hieraus viel geringere meridionale Temperatur-Gradienten und damit eine wesentliche Abschwächung der Westdrift um 20-30\% (FLOHN 1959b), deren Auswirkungen sich sicher bis in die Subtropen, ja Randtropen hinein erstrecken. Fragestellungen dieser Art, die sich nicht mehr mittels aktualistischer Analogien beantworten lassen, braucht man heute nicht mehr der ungezügelten Phantasie populärer Autoren zu überlassen: sie sind rationalen, physikalisch-mathematisch fundierten Erwägungen und Modellrechnungen zugänglich.

Auf die Analogien zwischen den für die Klimaschwankungen verantwortlichen Anomalien der atmosphärischen Großzirkulation in rezenten, historischen und pleistozänen Zeitabschnitten hat in den letzten Jahren vor allem H. H. LАмв $(1959,1961)$ hingewiesen. Im folgenden sollen einige (keinesfalls neue) Gedankengänge zur meteorologisch-klimatologischen Interpretation der pleistozänen Klimaschwankungen erörtert werden. $\mathrm{Da}$ es dem Verfasser nicht möglich ist, die umfangreiche geologische Literatur durchzusehen und im einzelnen zu zitieren (vg!. Woldstedt 1961), stellt er diese Gedankengänge nur in kurzer Form zur Diskussion. Natürlich kann die Aufgabe des Meteorologen hier nur in einer Interpretation geologischer Befunde bestehen. Die Anwendung moderner physikalischer Techniken - insbesondere Datierung und Temperaturbestimmung mittels radioaktiver oder stabiler Isotope - liefert uns heute quantitativ auswertbare Daten, bei deren Interpretation jedoch mit aller methodischen Kritik und Sorgfalt vorgegangen werden muß.

\section{Sind Pluviale und Eiszeiten synchron?}

In den letzten Tahrzehnten ist es meist üblich, die Pluvialzeiten in den subtropischen und tropischen Trockengebieten der nördlichen Halbkugel zeitlich den Eiszeiten (Glazialen) gleichzusetzen. Hieraus eroibt sich bei vielen Autoren die Auffassung, daß während der Eiszeiten die Trockengürtel der Erde von beiden Seiten her eingeengt wurden. Tatsächlich liegt aber in vielen Gebieten eine eindeutige Datierung pluvialer Ablagerungen noch nicht vor, so daß die Auffassung der Gleichzeitigkeit nur zu häufig lediglich auf Analogieschlüssen beruht.

In diesem Zusammenhang ist vielfach (so auch FLOHN 1952) die Hypothese vertreten worden, daß während der Eiszeiten mit ihrer gesteigerten Meridionalzirkulation - die auch den oben erwähnten 'Typ des , high index in n i ed rig e n Breiten " charakterisiert der subtropische Hochdruckgürtel abgeschwächt und die trovisch-äquatorialen Regen weiter verbreitet waren. Eine solche Korrelation ergibt sich z. B. im indischen Subkontinent aus dem Auftreten intensiver tropischer (zyklonaler) Störungen im Zusammenhang mit Höhentrögen der Westdrift, die auf deren Vorderseite weit polwärts ausgreifen. Demgegenüber vertrat W WLLETT (1950) den Standpunkt, daß in Zeiten gesteigerter Meridionalzirkulation (low index) der subtropische Hochdruckgürtel als Ganzes nur äquatorwärts verschoben sei. 
Diese Frage hat inzwischen eine neue Beleuchtung erfahren. Es scheint gesichert zu sein - vgl. Butzer 1958 - daß in weiten Gebieten des Nahen Ostens und Nordafrikas in der postglazialen Wärmezeit, also etwa 3-5000 v. Chr., pluviale Bedingungen herrschten. Dies war für den Meteorologen mit den bisherigen Kenntnissen von der atmosphärischen Zirkulation schwer zu vereinbaren, da bei einem verstärkten, polwärts verschobenen Subtropenhoch - wie es zur Erklärung der postglazialen Wärmezeit angenommen werden muß - die eben erwähnte Korrelation Höhentrog - tropische Störung nicht häufiger, sondern seltener als heute auftreten müßte. Inzwischen ergibt sich eine Erklärung für diese Diskrepanz in folgenden, noch wenig bekannten meteorologischen Erfahrungen:

In Nordafrika kommt es heute nicht ganz selten im Spätsommer zur Verlagerung ursprünglich tropischer Störungen bis nach Ägypten hin (mehrere Einzelfälle in den Monaten August bis Oktober sind inzwischen beschrieben worden), die mit ihren Wolkenfeldern und mit einzelnen Niederschlägen bis $25-27^{\circ} \mathrm{N}$, ja bis an die ägyptische Mittelmeerküste ausgreifen können (KocH, Abd el-Hady). Damit werden auch die eigenartigen Klimanotizen aus Alexandria in der Ptolemäerzeit (Ввоокs, S. $333 \mathrm{f}$ ) verständlich, die (ähnlich wie heute etwa im Raume von Quetta/Peshawar) von einer zweiten sommerlichen Regenzeit mit Gewittern neben der heute dominierenden Winterregenzeit berichten. Nach diesen Befunden können also auch in Perioden mit ausgeprägter Zonalzirkulation in hohen Breiten, also bei einem polwärts verschobenen Subtropenhoch, äquatoriale Störungen bis in subtropische Breiten $\left(28-32^{\circ} \mathrm{N}\right.$ ) vordringen. Damit wären die Befunde (Butzer 1958 u. a.) einer feuchten Periode in Nordafrika und dem Nahen Osten während der postglazialen Wärmezeit im aktualistischen Sinne meteorologisch deutbar; wenige Fälle dieser Art pro Jahr können genügen, um die mittlere Regenmenge um vielleicht $100 \mathrm{~mm}$ zu erhöhen, mit tiefgreifenden Folgen für Vegetation, Tierwelt und Hydrologie.

Im Lichte dieser Erkenntnis gewinnen die Einwände (Balout 1952, Schwarzbach 1953) gegen die Gleichzeitigkeit von Pluvial und Glazial ein neues Gesicht (vgl. auch BüDEL 1963). Man wird in Zukunft klar unterscheiden müssen zwischen einem „pol a re n " P1 uvia 1, das in erster Linie auf die mit der "Polarfront" - richtiger: mit den wetterwirksamen Mechanismen der außertropischen Westdrift — zusammenhängenden, äquatorwärts ausgreifenden Winterregen zurückgeht und gleichzeitig mit den Eiszeiten auftritt, und einem tropischen Pluvialli), das umgekehrt in den „interglazialen" Perioden bei einem verstärkten, nach Norden verschobenen Subtropenhoch mit vorwiegend tropischen Sommerregen auftritt. Beide können in den gleichen Räumen und in den gleichen Zeitabschnitten vorkommen, ebenso wie das z. Zt. für extreme Wetterlagen gilt. Hiernach ist es zweifellos nicht mehr erlaubt, pluvial-zeitliche Ablagerungen in der heutigen Trockenzone ohne weitere Untersuchungen mit den Eiszeiten zu korrelieren. Nur mit einer einwandfreien Datierung mit Hilfe moderner Verfahren kann man schwerwiegende Fehlschlüsse vermeiden; vielleicht ist es sogar möglich, einmal mit exakten Temperaturbestimmungen bei der Bodenbildung zwischen Winterund Sommerregen zu unterscheiden. Andererseits ist es notwendig, von meteorologischen Gesichtspunkten aus Intensität und Ausdehnung der (aktuellen) Niederschläge in verschiedenen Teilen der Tropenzone in ihrem Zusammenhang mit den Zirkulationstypen der Westdrift zu untersuchen. Erst eine solche (aktualistische) Untersuchung kann die Zusammenhänge zwischen den alternierenden Pluvialen der Tropen- und Subtropenzone und den extratropischen Glazialen klären.

1) Verfasser schlägt diese beiden Begriffe vor, da die von BALout und seinem meteorologischen Gewährsmann DubIEF - dem besten Kenner des Sahara-Klimas - gebrauchten Begriffe „etesische Winde" und "Monsune" hier etwas mißverständlich in erweitertem Sinne gebraucht werden: die Etesien sind sommerliche trocke ne N-winde im Mittelmeergebiet, und der den ganzen afrikanischen Kontinent durchsetzende "Monsun" weht in seinem Kerngebiet beiderseits des Äquators ganzjährig aus SW. 


\section{Zur Frage des eustatischen Absinkens des Weltmeeres in den Interglazialzeiten}

Die eustatischen Meeresspiegelschwankungen zwischen den Eiszeiten einerseits, den Interglazialzeiten (einschließlich Postglazial) andererseits werden allgemein als Folge des Umsatzes von Wasser in fester bzw. flüssiger Form zwischen den beiden großen Speichern - dem Weltmeer und den kontinentalen Eisschilden - gedeutet. In diesem Sinn bilden die eustatischen Schwankungen (FAIRBRIDGE 1961a, 1961b, Graul 1959) global integrierte Maßgrößen der kontinentalen Vereisung und erlauben einige quantitative Abschätzungen. Hierbei dürfen wir aber nicht ohne weitere Überlegungen die auf dem Festland gespeicherte Eismasse mit der dem Meere entzogenen Wassermenge gleichsetzen. Dies setzt zunächst voraus, daß die Speicherung von Wasserdampf in der Luft (heute entsprechend 2,4 cm Niederschlagswasser) vernachlässigbar gering ist und daß auch die globale Jahresmenge von Niederschlag und Verdunstung nur untergeordneten Schwankungen unterliegt. Diese ist wegen der prinzipiellen Schwierigkeiten der Messung auf den weiten Ozeanflächen nur ungenau bekannt: sie beträgt nach der Mehrzahl der „westlichen“ Autoren $81-86 \mathrm{~cm} / \mathrm{a}$, nach Budyko dagegen $93 \mathrm{~cm} / \mathrm{a}$. Damit verbleibt jedes Wasserdampfmolekül im Mittel nur 11-13 Tage in der Atmosphäre, dagegen etwa 4000 Jahre im Ozean. Eine Abschätzung der Verweildauer im antarktischen Inlandeis ist weit unsicherer: ein großer Teil des Niederschlags wird durch Schneefegen und Gletscherbewegung wieder relativ rasch entfernt, während im Innern die sehr kalten (ca. $-28^{\circ} \mathrm{C}$ ) Eismassen nur sehr geringe Bewegungen ausführen; ein Mittelwert von mindestens 20000 Jahren sagt also hier wenig aus. Ferner haben wir hier die Änderungen in der horizontalen Ausdehnung des Weltmeeres zu berücksichtigen, wobei das Trockenfallen weiter Schelfmeere sowie die Überflutung weiter Tiefländer dringend einer genauen quantitativen Abschätzung bedarf. Ein weiterer, nicht ganz zu vernachlässigender Faktor ist die Dichteänderung des Meerwassers mit der Temperatur: bei einem mittleren thermischen Ausdehnungskoeffizienten von 0,00014 und einer mittleren Ozeantiefe von $3800 \mathrm{~m}$ liefert eine Temperaturabnahme von $5^{\circ}$ eine Senkung des Meeresspiegels um 2,66 m.

Eine der auffallendsten Erscheinungen ist das anscheinend kontinuierliche (ZEUNER, FaIRbridge 1961a) eustatische Absinken der interglazialen Meeress pieg e $1 \mathrm{~s}$ tände um mindestens $80 \mathrm{~m}$ seit dem Sizilian, also in einem Zeitraum, der nach der älteren Zeitskala sich auf etwa $650000 \alpha$ beläuft. Dieses Absinken entspricht einer mittleren jährlichen Spiegeländerung um -0,12 mm/a. Die Deutung dieses Vorganges wird meist auf eine langsame Senkung des Meeresbodens etwa im Pazifik zurückgeführt (Tektono-Eustasie). Für eine solche Senkung gibt es zwar - in den flachen Oberflächen der unterseeischen Guyots - Anhaltspunkte, aber ihr Betrag und ihre zeitliche Stellung sind noch weitgehend hypothetisch.

Eine andere, bisher offenbar vernachlässigte Modellvorstellung geht vom Aufbau des antarktischen Eises aus. Wir können uns vorstellen, daß etwa im Pliozän nach Ausweis der paläomagnetischen Daten der antarktische Kontinent in die Nähe des Südpols gedriftet war und sich dann - vielleicht gerade an der Wende Plio/Pleistozän - langsam eine flache Eiskappe bildete (FAIRBRIDCE 1961b), die zunächst noch lange unter den Bedingungen eines „temperierten“ Gletschers (rasche Bewegung, sommerliches Abschmelzen der Oberfläche bei enormer Einstrahlung, einer Albedo $<50 \%$ und Temperaturen $>0^{\circ}$ ) mit etwa stationärer Eisbildung verharrte. Die Bedeutung dieses Unterschiedes zwischen temperierten und kalten Gletschern für den Eishaushalt von Inlandeisgebieten hat besonders Hoinkes (1961) herausgestellt. Damit ging ein allmähliches Absinken der Meerestemperatur einher, wie es durch die Tiefsee-Bohrkerne belegt ist (EmiLiani 1961). Aber erst im Verlauf des ältesten Pleistozäns kam es bei allgemeiner Abkühlung - über deren Ursache hier keine Spekulationen angestellt werden sollen - zu einem Absinken der Sommertemperaturen unter $0^{\circ}$ und zu den Bedingungen eines „kalten“ Gletschers (geringe Be- 
wegung, Temperaturen ständig $<0^{\circ}$, Albedo $85-90 \%$ ). Da nunmehr die Summe der Niederschläge größer war als die Summe aller Verluste, wurde jetzt die Eisbildung positiv. Das antarktische Inlandeis - das während des Stadiums des warmen Gletschers vielleicht nur im Mittel $300 \mathrm{~m}$ mächtig war (entsprechend einer Eismasse von etwa $4.10^{6} \mathrm{~km}^{3}$ und einem Absinken des Weltmeeres um knapp $10 \mathrm{~m}$ ) - wuchs nunmehr unter Schwankungen an. Wenn wir diese Oszillationen vernachlässigen und für den Aufbau des AntarktisEises einen Mittelwert für den gesamten Zeitraum seit dem Sizilian (Günz/Mindel-Interglazial oder Vor-Günz?) annehmen, dann entspricht die mittlere Meeresspiegeländerung von $-0,12 \mathrm{~mm} / \mathrm{a}$ einem Anwachsen des antarktischen Eises um 3,8 mm/a. Dieser Wert wäre zu vergleichen mit dem heutigen Wert von mindestens $+2 \mathrm{~cm} / \mathrm{a}$ (HoINkEs 1961). Diese Zahlen stimmen der Größenordnung nach befriedigend überein, besonders wenn man bedenkt, daß der Aufbau des antarktischen Eises erheblichen zeitlichen Schwankungen unterworfen war. Dabei darf sicher nicht vorausgesetzt werden, daß diese Schwankungen mit dem Wechsel zwischen Eiszeit und Interglazial auf der Nordhemisphäre synchron waren. Seit R. F. Scott und Meinardus haben viele Autoren - so Büdel (1960) - auf die Wahrscheinlichkeit einer Gegenläufigkeit - Wachstum in den relativ warmen Interglazialen, Stillstand oder Abbau in den Eiszeiten - hingewiesen. Andererseits war zu einem noch nicht festlegbaren Zeitpunkt das antarktische Inlandeis mindestens $300 \mathrm{~m}$ mächtiger als heute (HoINkes 1961), was einem Massenverlust von etwa 15\% entspricht. Wenn wir das heutige Anwachsen von $2 \mathrm{~cm}$ /a auch als repräsentativen Wert für einen Rückgang veranschlagen - das ist vermutlich ein Höchstwert! - , dann müßte dieses Maximum mindestens $15000 \alpha$ zurückliegen. Vielleicht darf man mit aller Reserve die Arbeitshypothese aussprechen, daß dieser Höchststand wohl kaum später gelegen haben kann als das R/W-Interglazial (Eem-Transgression), jedenfalls nicht im Postglazial.

Nehmen wir den heute wohl besten Wert von $28.10^{6} \mathrm{~km}^{3}$ für die mittlere Masse des antarktischen Eises (Hoinkes 1961), dann entspricht die Wassermenge von $25,2.1015 \mathrm{~m}^{3}$ (hier einschließlich des oben vorausgesetzten flachen pliozänen Eisschildes) einem Meeresspiegelanstieg von rund $70 \mathrm{~m}$. Wenn wir eine Ausdehnung der Meeresfläche durch Überflutung der weiten Niederungslandschaften um nur $4 \%\left(=14,5.10^{6} \mathrm{~km}^{2}\right)$ voraussetzen, reduziert sich der Anstieg auf $\left.66 \mathrm{~m} .^{2}\right)$ Das reicht nicht ganz aus, um das beobachtete eustatische Absinken seit dem Sizilian zu erklären. Aber zweifellos ist das antarktische Festland (ebenso wie Grönland) durch die Eisbelastung erheblich unter den Meeresspiegel gedrückt worden. Größenordnung und Vorzeichen der hierdurch veranlaßten isostatischen Spiegelschwankungen lassen sich nur hypothetisch abschätzen, können aber zur Erklärung der noch verbleibenden Differenz herangezogen werden.

Gegenüber allen großtektonischen Hypothesen besitzt diese glazial-e ustatische $\mathrm{Hy}$ p othes e der gesamten pleistozänen Schwankungen des Meeresspiegels (einschließlich des interglazialen Absinkens) manche Vorzüge, vor allem den der Einheitlichkeit. Sie setzt voraus, daß im Gebiet der Antarktis seit Beginn des Pleistozäns physikalischklimatische Bedingungen herrschten, die mit nur graduellen Schwankungen den heutigen etwa gleichkamen, aber prinzipiell von denen des gesamten Tertiärs abwichen. In diesem Zusammenhang wäre es wichtig zu klären, seit wann das grönländische Inlandeis existiert und ob es - was trotz seiner viel geringeren Ausdehnung wahrscheinlich erscheint - eine oder mehrere Warmzeiten überdauert hat.

Aus dieser Hypothese ergeben sich noch einige wichtige Folgerungen für den großräumigen Klimaablauf während des Pleistozäns. Wenn das antarktische Inlandeis bereits

2) $\mathrm{Zu}$ diesen Mengen muß man - worauf Prof. WoLDstedt den Verfasser mit vollem Recht hinwies - die im Inlandeis Grönlands festgelegte Wassermenge hinzurechnen, die sich nach den heute besten Abschätzungen auf etwa $2,5.1015 \mathrm{~m}^{3}$ beläuft; dann kommen wir auf einen Meeresstand im Sizilian von rund +76 bzw. $72 \mathrm{~m}$. 
im ältesten Pleistozän (d.h. vor der Günz-Vereisung) existierte, wenn auch nur als „temperierter Gletscher", dann genügte dies zu einer erheblichen Zunahme der südhemisphärischen Westdrift (FLOHN 1959b) um vielleicht 50\% gegenüber einem hypothetischen „akryogenen " Zustand. Während im eisfreien Tertiär und Mesozoikum die tropisch-subtropischen Wind- und Klimagürtel - wegen des erheblich geringeren meridionalen Temperaturgradienten - offenbar merklich weiter polwärts ausgedehnt waren als heute und die subpolare Tiefdruckzone sich auf die Polarkappen beschränkte, kam es jetzt zu einer Einengung und Verschiebung dieser Klimagürtel in Richtung auf den Äquator. Wenn das antarktische Inlandeis sich (wegen der polnäheren Lage des antarktischen Festlandes) früher gebildet hat als alle Eiszentren der Nordhemisphäre, dann tauchte damals in ganz ausgeprägter Form erstmals ein sehr charakteristisches Phänomen auf: eine Asymmetrie der tropischen Zirkulationsgürtel in Bezug auf den Äquator, wobei die viel intensivere Westdrift der Südhemisphäre den meteorologischen Äquator ganzjährig auf die Nordhalbkugel drängt. Eine derartige äquatoriale Asymmetrie existiert auch heute (z. B. FloHN 1959a), wenn auch in schwächerem Ausmaß; heute liegt der meteorologische Äquator im Jahresmittel in $5-7^{\circ} \mathrm{N}$, und über dem Meereis der Arktis ist die Troposphäre im Winter etwa $5^{\circ}$, im Sommer etwa $10^{\circ}$ wärmer als über der Antarktis. Die äquatoriale Asymmetrie wurde verringert, nachdem sich - möglicherweise mit beträchtlicher Verzögerung gegenüber der Antarktis, aber sicher früher als das laurentische oder das skandinavische Eis - zum ersten Mal eine ausgedehnte Vereisung von Grönland und dem Barentsmeer-Schelf (BüDEL 1963) gebildet hatte; damit verstärkte sich (wenigstens in den Kaltzeiten) die nordhemisphärische Westdrift in ähnlicher Weise wie die der Südhalbkugel. Diese Asymmetrie des meteorologischen Aquators erreichte vermutlich in den Warmzeiten des ältesten und älteren Pleistozäns ihren Höhepunkt, dagegen in den letzten Kaltzeiten (Riß, Würm) ihr Minimum. Diese aus meteorologischen Gründen zu erwartenden Vorgänge haben sehr weitreichende Konsequenzen für die Klimageschichte der nicht vereisten tropisch-subtropischen Zonen, die sich jedoch erst nach einer einwandfreien, weltweit vergleichenden Datierung richtig abschätzen lassen. Es handelt sich dabei um Verlagerungen und Ausweitungen der äquatorialen Regengebiete und der subtropischen Trockengebiete mit ihren jahreszeitlich alternierenden Übergangsgürteln der tropischen Sommerregenzone und der subtropischen Winterregenzone.

\section{Eustatischer Spiegelanstieg im Spät- und Postglazial und Wärmehaushalt}

Das Abschmelzen der großen Inlandeisgebiete von Nordeuropa und Nordamerika während der Spät- und Postglazialzeit verlangt zunächst eine völlige Umordnung der atmosphärischen Zirkulation, ähnlich derjenigen, welche den Umbruch von einer strengwinterlichen Wetterlage zu verbreitetem Tauwetter herbeiführt. Diese Umordnung verlangt eine einschneidende Änderung im Wärmehaushalt, obwohl die Anhäufung von Schnee und Eis mit der zugehörigen hohen Albedo und langwelligen Ausstrahlung, mit der kräftigen Produktion katabatisch abfließender Kaltluft zunächst noch kaum verändert fortbesteht. Da die maximal möglichen Änderungen der Einstrahlung - nach der astronomischen Theorie der Klimaänderungen - nur äußerst langsam vor sich gehen und nur wenige Prozent betragen, muß der folgenschwere Umbruch der Großwetterlage weitgehend advektiver Natur sein, d. h. im Zusammenhang stehen mit meteorologisch-klimatologischen Vorgängen in dem immer unvereisten größten Teil der Erdoberfläche. Die kontinentalen Eisschilde (einschließlich der Antarktis) nahmen maximal $45.10^{6} \mathrm{~km}^{2}=8,8 \%$ der Erdoberfläche ein - gegenüber heute rund $15.10^{6} \mathrm{~km}^{2}$ oder rund $3 \%$ - die meereisbedeckten Ozeane vielleicht weitere $30.16^{6} \mathrm{~km}^{2}$. Damit standen insgesamt etwa $15 \%$ der Erdoberfläche unmittelbar unter „kryogenem “ Klimaeinfluß, aber immer noch 85\% insbesondere die gesamte Tropen- und Subtropenzone! - unter den normalen Bedingungen eines „akryogenen“ Wärmehaushalts. 
Welche Anforderungen stellte nun das Abschmelzen der nordhemisphärischen Inlandeismassen im Postglazial an den globalen Wärmehaushalt? Nach FAIRBRIDGE (1961a) spielte sich ein starker Meeresspiegelanstieg zwischen der Jüngeren Dryas-Zeit (ValdersStadium, ca. 8400 v. Chr.) und dem Beginn des postglazialen Wärmeoptimums (ältere Peron-Transgression, ca. 3800 v. Chr.) ab: hier wurde in $4600^{a}$ ein Ansteigen um volle $42 \mathrm{~m}$ beobachtet, d. h. um rund $9 \mathrm{~mm} / \mathrm{a}$. Dieser Anstieg konzentrierte sich besonders auf die Zeit vom Valders-Stadium bis zum Beginn des Cochrane-Vorstoßes (entsprechend dem Yoldia-Stadium des Ostseegebietes, ca. 6600 v. Chr.). In dieser Zeit müssen wir nach den übereinstimmenden Angaben von Graul (1959) und FaIRBRIDGe (1961a) mit einem Anstieg um etwa $27 \mathrm{~m}$ in rund 1800 Jahren rechnen, d. h. um etwa $15 \mathrm{~mm} / \mathrm{a}$. Wir können für diese relativ kurze Zeitspanne einmal versuchsweise für die Antarktis eine stationäre Eisbilanz annehmen, da sich aus dem Eisrückgang dort die Annahme einer (wie heute) positiven Eisbilanz verbietet.

Wenn wir nun die kleineren Eiszentren vernachlässigen, entspricht dieser Spiegelanstieg von $15 \mathrm{~mm} / \mathrm{a}$ für die Hauptvereisungszentren Nordamerika und Nordeuropa deren Fläche sich gegenüber dem Höchststand (vgl. GRAuL) um ca. 15\% verringert hatte einem Abschmelzverlust von etwa $24 \mathrm{~cm} / \mathrm{a}$. Dieser Wert erscheint nicht unvernünftig, wenn wir ihn mit dem rezenten Schrumpfen der isolierten Ostalpengletscher von $60 \mathrm{~cm} / \mathrm{a}$ vergleichen. Ein jährliches Abschmelzen von $21,6 \mathrm{~cm}$ Wasseräquivalent erfordert rund $1730 \mathrm{gcal} / \mathrm{cm}^{2}$; das wären im Jahresmittel nur knapp 1\% der (globalen) extraterrestrischen Einstrahlung auf eine horizontale Fläche, im kurzen Sommer (auf den sich das Abschmelzen konzentriert) allerdings etwas mehr. Es wäre eine interessante Aufgabe, nach unseren heutigen Kenntnissen über den Wärme- und Massenhaushalt von Gletschern - der sich in den einzelnen Klimazonen ganz verschiedenartig verhält! - einmal diese Größen für die Inlandeismassen der Postglazialzeit abzuschätzen. Dabei spielt die hohe Albedo des Firneises bekanntlich eine ausschlaggebende Rolle; die effektive Einstrahlung ist im Jahresdurchschnitt unter diesen Umständen nur geringfügig höher als die effektive Ausstrahlung. Einige Überlegungen (s. Einleitung) führen zu der Auffassung, daß während des Sommers der abschmelzende Sektor des Inlandeises vermutlich meist (auch wegen des starken Wärmeverbrauchs) wolken- oder nebelbedeckt war, so daß ein beträchtlicher Teil der benötigten Energie durch advektive Prozesse - Wärmetransport von der herangeführten Warmluft zum Gletscher, fallender Regen — gedeckt werden mußte. Da aber der globale Strahlungshaushalt ein Vielfaches dieser Energiebeträge liefert, ist das ohne weiteres möglich. Selbst der von FAIRBRIDGE angegebene Extremwert des eustatischen Anstiegs von $50 \mathrm{~mm} / \mathrm{a}$ entsprechend einem Abschmelzen von rund $80 \mathrm{~cm} / \mathrm{a}$ Eis erscheint vom energetischen Standpunkt aus noch vertretbar. Da sich das Abschmelzen auf wenige Sommermonate konzentriert, wird die sommerliche Abflußmenge im Gebietsmittel sehr groß; hinzu kommt die Konzentration des Abflusses in den Randgebieten, die zur Ausbildung von Riesenströmen führt. Um ein Beispiel zu geben: setzen wir gegen Ende des Hochglazials für die untere Elbe ein Einzugsgebiet (einschließlich der Abdachung des Inlandeises) von nur etwa $2.10^{6} \mathrm{~km}^{2}$ an sowie im Sommer (5 Monate Mai-September) räumliche Mittelwerte für Niederschlag $=250 \mathrm{~mm}$, Verdunstung $=225 \mathrm{~mm}$ und Eisschmelze $=125 \mathrm{~mm}$, d. h. eine Abflußspende von $11 / \mathrm{m}^{2} \mathrm{~d}$, dann liefert das einen $A b f l u ß$ von rund $23000 \mathrm{~m}^{3} / \mathrm{sec}$, d. h. etwa 60\% der Abflußmenge im Unterlauf des Kongo oder 20\% der des Amazonas (KELLER 1961). Natürlich kann diese durch subglazialen Abfluß im Ostseegebiet verringert werden.

\section{Liter a tur}

Abd EL-Hady, S. S.: Vortrag WMO-ICAO-Seminar Cairo, Dez. 1961.

BALOut, L.: Pluviaux interglaciaires et préhistoire saharienne. - Trav. Inst. Rech. Sahar. 8, 9-22, Alger 1952.

Brooks, C. E. P.: Climate through the Ages. - 2. edit.: London 1949. 
BüDEL, J.: Die Gliederung der Würmkaltzeit. - - Würzburger geogr. Arb. 8, Würzburg 1960. - Die jungpliozänen und quartären „Pluvialzeiten“ der Sahara. - Eiszeitalter u. Gegenwart 13, Ohringen/Württ. 1963.

Butzer, K. W.: Quarternary Stratigraphy and Climate in the Near East. - Bonner geogr. Abh. 24, Bonn 1958.

Emiliani, C.: Cenozoic Climate Changes as indicated by the Stratigraphy and Chronology of Deep-Sea Cores of Globigerina-Ooze Fazies. - Ann. New York Acad. Sci. 95, 521-536, New York 1961.

FAIrbridge, R. W.: Eustatic Changes in Sea Level. - Physics and Chemistry of the Earth 4, 99-185, London 1961(a). - - Convergence of Evidence on Climatic Changes and IceAges. - Ann. New York Acad. Sci. 95, 542-579, New York 1961(b).

FLohn, H.: Atmosphärische Zirkulation und Paläoklimatologie. - Geol. Rundsch. 40, 153-178, Stuttgart 1952. - - Prob!eme der geophysikalisch-vergleichenden Klimatologie seit Alexander von Humboldt. - Ber. dt. Wetterdienst 59, 9-31, Offenbach 1959(a). - - Kontinental-Verschiebungen, Polwanderungen und Vorzeitklimate im Lichte paläomagnetischer Meßergebnisse. - Naturwiss. Rundsch. 1959, 375-384, Frankfurt 1959(b).

Graul, H.: Der Verlauf des glazialeustatischen Meeresspiegelanstieges, berechnet an Hand von Cl4-Datierungen. - Wiss. Abh. dt. Geographentag 1959, 232-242, Wiesbaden 1960.

Hoinkes, H.: Die Antarktis und die geophysikalische Erforschung der Erde. - Naturwiss. 48, 354-374, Berlin 1961.

Keller, R.: Gewässer und Wasserhaushalt des Festlandes. - Berlin 1961.

Kосн, H. G.: Meteorologische Studien im Mittelmeer. - Abh. Met. Hydr. Dienst DDR 1 , Berlin 1950.

LAMB, H. H.: On the nature of certain climatic epochs which differed from the modern (1900-39) normal. In: Changes of Climate, Unesco-WMO Symposium Rome 1961, Arid Zone Research Vol. 20, 125-150.

Lamb, H. H. \& Johnson, A. J.: Climatic Variations and Observed Changes in the General Circulation. - Geogr. Ann. 41, 94-134, Stockholm 1959 und 43, 363-400, 1961.

Schwarzbach, M.: Das Alter der Wüste Sahara. - N. Jb. Geol. Paläont., Mh., 157-174, Stuttgart 1953.

Willetr, H. C.: The General Circulation at the last (Würm) Glacial Maximum. - Geogr. Ann. 31, 179-187, Stockholm 1950.

Willett, H. C. \& SAnDERS, F.: Descriptive Meteorology. - 2. edit., New York 1959.

Woldstedt, P.: Das Eiszeitalter. Grundlinien einer Geologie des Quartärs. - 3. Aufl., Stuttgart 1961

Zeuner, F. E.: Dating the Past. - 4. edit., London 1959.

Manuskr. eingeg. 23. 2. 1963.

Anschrift des Verf.: Prof. Dr. Hermann Flohn, Meteorol. Inst. der Universität, Bonn, Kurfürstenstraße 74. 